\title{
Markers of Atherosclerosis in Hypertensive Patients with Less Advanced Chronic Kidney Disease
}

\author{
Claudia Floriana Suciu\#, Andreea Varga"\# ${ }^{\star}$, Corneliu Florin Buicu, Ioan Tilea \\ University of Medicine, Pharmacy, Science and Technology of Targu Mures, Romania
}

\begin{abstract}
Objective: Our study aimed to validate the neutrophil-to-lymphocyte ratio (NLR) as a marker for aortic arch calcification in hypertensive patients with less advanced chronic kidney disease (CKD). Methods: A number of forty-four hypertensive patients with chronic kidney disease (categories G3a and G3b - 2012 KDIGO nomenclature) were included in the study. Considering the presence of aortic arch calcification (AAC) on chest X-ray, the study population was divided into two groups: 27 patients AAC present and seventeen without aortic arch calcification. Laboratory data were collected for each patient and NLR was computed. Comorbidities were also recorded: stable coronary artery disease, lower extremity arterial disease and hypertensive heart disease. Results: A positive correlation between neutrophil-to-lymphocyte ratio and aortic arch calcification in hypertensive CKD patients was identified. Furthermore, advanced age, increased alkaline phosphatase and increased erythrocyte sedimentation rate had a positive association with aortic arch calcification. We found no statistical correlation between neutrophil-to-lymphocyte ratio and other laboratory features in both groups of patients. Conclusions: Neutrophil-to-lymphocyte ratio may be viewed as a potential risk factor for vascular calcification in patients with moderate chronic kidney disease; nevertheless, future extensive studies are necessary. In the management of hypertensive patients, general medicine might particularly benefit of this simple, readily available inflammatory marker.
\end{abstract}

Keywords: neutrophil-to-lymphocyte ratio, atherosclerosis, systemic hypertension, chronic kidney disease, aortic arch calcification

Received 03 August 2019 / Accepted 22 August 2019

\section{Introduction}

Inflammatory markers tend to increase with age, and additionally, in the elderly population, inflammation is acknowledged as an essential risk factor for morbidity and mortality [1].

Chronic inflammation has been reported as an important prognostic factor for chronic kidney disease (CKD) progression; a high prevalence of it has been long documented in individuals with CKD, particularly in endstage renal disease patients [2]. Currently, serum markers of inflammation are recognised as potential indicators for early diagnosis, prognosis, and CKD follow-up. C-reactive protein (CRP), interleukin- 6 and tumoral necrosis factoralpha determine injurious effects on the mesangial and endothelial glomerular cells by increasing the production of the extracellular matrix. Moreover, they also lead to degradation and reduction of the stimulated extracellular matrix, hence resulting in renal function decline due to glomerular hypertension, tubulointerstitial fibrosis and renal scarring $[3,4]$. In haemodialysis patients, CRP remains the most relevant inflammation biomarker, accepted as a validated cardiovascular morbi-mortality prognostic factor [5]. A novel biomarker of inflammation - pentraxin 3 is also associated with CKD progression and may be more sensitive in the prediction of cardiovascular mortality compared to high-sensitive CRP [6-8].

The neutrophil-to-lymphocyte ratio (NLR), recently recognised as an inflammation marker, is an emerging prognostic factor for cancer, cardiovascular diseases [9-13].

* Correspondence to: Andreea Varga

E-mail: dr.andreeavarga@gmail.com

\# These authors have equal contribution to the article.
No worldwide reference values are validated; European, Asian and Latin-American cohort studies describe different values related to age, gender. In healthy adults, aged under 66 years old, Forget and colab. reported normal NLR values between 0.78 to 3.53 [9].

Inflammation plays an essential role in the pathomechanisms of atherosclerotic disease; additionally, NLR is recognised as a predictor of subclinical and clinical atherosclerotic disease $[14,15]$. Recent data suggest that NLR may be an indicator for the prevalence and severity of coronary artery disease and also for the extent of aortic arch calcification (AAC) $[16,17]$. In CKD patients, neutrophil-tolymphocyte ratio, an inexpensive and convenient available inflammatory marker has also emerged as a prognostic factor for adverse renal events. In the Asian population, NLR is associated with the risk of progression towards end-stage renal disease $[18,19]$.

Vascular calcification, a common finding in CKD patients, is a result of two independent mechanisms. One process, represented by atherosclerotic calcification, is characterized by plaque formation with patchy localisation at the intimal layer followed by calcification induced by osteoblast stimulation; atherosclerotic calcification is related to traditional risk factors for vascular calcification: arterial hypertension, dyslipidemia, smoking, ageing and diabetes mellitus. The second pattern described as arteriosclerosis is characterised by medial artery calcification, as a consequence of non-traditional risk factors for vascular calcification such as hyperphosphatemia, hyperparathyroidism and inappropriate cytokines expression.

Arteriosclerosis is common in patients with chronic kidney disease and diabetes [20]. The extent and type of 
vascular calcifications are acknowledged as mortality predictors due to vascular complications [21,22].

Aortic arch calcification, identified on conventional posteroanterior chest X-ray, has been recognised as a reliable, independent predictor of cardiovascular events beyond traditional risk factors in CKD patients but also in the general population $[23,24]$.

Moreover, hypertension alone can promote medial calcification by complex mechanisms which involve increased arterial stiffness. In CKD patients these has paramount implications as hypertension has a frequent occurrence and may lead to premature arterial calcification [25].

Association between NLR and AAC, debated in a large number of papers, emphasized neutrophil-to-lymphocyte ratio as a predictor of arterial calcification extent [26]. On the other hand, the neutrophil-to-lymphocyte ratio proved to be a prognostic factor for vascular calcification in patients with end-stage renal disease as well [22,27].

Both NLR and AAC are associated with arterial stiffness, as neutrophil-to-lymphocyte ratio is independently associated with arterial stiffness in patients with advanced kidney disease, and aortic calcification is associated with arterial stiffness in the general population and hypertensive patients [28-30].

In the present study, we aimed to validate the neutrophil-to-lymphocyte ratio as a marker for aortic arch calcification in hypertensive non end-stage CKD patients.

\section{Methods}

Two-hundred eighty electronic patient medical records, enrolled between January 2018 to June 2019, were reviewed and analysed. Main inclusion criteria were represented by the diagnosis of hypertension, chronic kidney disease and availability of posteroanterior chest X-ray in Caucasian patients. Patients with type I and II diabetes mellitus, chronic obstructive pulmonary disease, benign prostatic hyperplasia, acute or chronic infections, cancers or other documented inflammatory conditions were excluded.

We identified 44 hypertensive CKD patients fulfilling inclusion criteria. Chronic kidney disease was diagnosed by the Kidney Disease Improving Global Outcomes (KDIGO) 2012 Clinical Practice Guideline for the Evaluation and Management of Chronic Kidney Disease, based on Glomerular Filtration Rate category criteria [31]. The diagnosis of hypertension was revised accordingly to the 2018 ESC/ESH Arterial Hypertension (Management of) Guidelines using the recorded office blood pressure measures [32]. For all patients, demographic and anthropometric data were collected following a detailed, standardized protocol.

The studied population was divided into two groups based on aortic arch calcification presence on chest X-ray: group 1 consisting of 27 hypertensive CKD patients in conjunction with AAC; group 2 included seventeen hypertensive $\mathrm{CKD}$ patients negative for AAC. Calcification of the aortic arch was estimated using the four grades de- scribed by Symeonidis et al: grade 0, defined by no sign of calcification; grade 1 - limited proof of calcification in the form of a thin calcification area or small spots; grade 2 - indicative of a more advanced calcification process with one or more extensive calcification areas; grade 3 - aortic knuckle circumferential calcification [33]. All patients enrolled in group 1 had a radiologic proof of aortic arch calcification grade 2 to 3 .

The following comorbidities were searched for: evidence of stable coronary artery disease, carotid stenosis, lower extremity arterial disease and hypertensive heart disease.

Presence of stable coronary artery disease (SCAD) was acknowledged in patients who met the 2013 ESC guidelines on the management of stable coronary artery disease, while lower extremity arterial disease (LEAD) was confirmed according to the 2017 ESC Guidelines on the Diagnosis and Treatment of Peripheral Arterial Diseases, in collaboration with the European Society for Vascular Surgery $[34,35]$. No history of carotid stenosis was documented in our study population.

For all forty-four patients, complete blood count with full automated differential counts (which included neutrophils and lymphocytes) and serum creatinine values were accessible. Neutrophil-to-lymphocyte ratio (expressed as number) was calculated by dividing the neutrophils count by the lymphocytes count. Estimated glomerular filtration rate (eGFR) was calculated based on the CKD Epidemiology Collaboration (CKD-EPI) formula. Where available, the following data were included in the descriptive and statistical analysis: erythrocyte sedimentation rate (ESR), fibrinogen, iron, total serum calcium, total cholesterol, triglyceride, alkaline phosphatase and uric acid. Due to the lack of availability other routine inflammatory markers (CRP, hs-CRP) were not analysed.

The data processing was carried out using GraphPad Prism 3.1 software (GraphPad Software Inc., San Diego, USA) and Statistical Package for Social Sciences (IBM Corp. Released 2013. IBM SPSS Statistics for Windows, Version 22.0. Armonk, NY, IBM Corp.). Continuous variables are expressed as mean \pm standard deviation (SD), and categorical variables are expressed as numbers. In order to assess the statistical significance of differences between the groups, we applied Student's t-test for parametric data; for non-parametric data the Mann-Whitney $U$ test was used. A p-value of $<0.05$ was considered statistically significant. Correlations between neutrophil-to-lymphocyte ratio and laboratory features were investigated through Spearman's rank correlation coefficient analysis. Regarding comorbidities and NLR association, the variations between the study groups were investigated by the non-parametric KruskalWallis test, and Dunn's multiple comparisons post-test.

This research regarding assessment of atherosclerotic disease in hypertensive patients was approved by the Committee on Medical Ethics, Targu Mures County Emergency Clinical Hospital no. 20874/2019. All procedures performed were in accordance with the ethical standards of 
the 1975 Declaration of Helsinki - Ethical Principles for Medical Research Involving Human Subjects, developed by World Medical Association and consequent amendments.

\section{Results}

The mean age for group 1 was $72.26 \pm 6.567$ years old. Out of 27 individuals from group 1, men represented $74.07 \%$. For group 2 the mean age was $65.82 \pm 5.399$ years old; men represented a total of $52.94 \%$ out of 17 individuals.

Baseline characteristics for study participants are illustrated in Table I.

With respect to $2012 \mathrm{KDIGO}$ classification, we included 36 patients in G3a category. Eight cases $(18.18 \%$ of all patients) were in G3b category. All G3b patients were part of group 1.

Table II depicts comorbidities distribution between the two groups.

Comparative data on age, iron, total cholesterol, triglyceride, ESR, fibrinogen, alkaline phosphatase and uric acid between the groups are displayed in table III. Aortic arch calcification positive patients had significantly higher values for ESR $(\mathrm{p}=0.034)$, eGFR $(\mathrm{p}=0.0007)$, total cholesterol $(\mathrm{p}=0.0074)$ and alkaline phosphatase $(\mathrm{p}=0.0074)$ compared to individuals without AAC. Age also proved to be higher in aortic arch calcification positive individuals (statistically strong significant, $\mathrm{p}=0.0001$ ). A statistically significant difference in other parameters presented in table III was not identified.

Comparative analyses regarding neutrophil-to-lymphocyte ratio distribution in the study population is depict- ed in figure 1. Aortic arch calcification is associated with increased values of neutrophil-to-lymphocyte ratio when compared with individuals without vascular calcification $(\mathrm{p}=0.007)$.

The correlations between NLR and collected laboratory data (alkaline phosphatase, uric acid, fibrinogen, total calcium, total cholesterol, triglycerides) are displayed in table IV and table V. Applying Spearman correlation analysis, we did not report any correlation between neutrophil-tolymphocyte ratio and laboratory values in aortic arch calcification positive patients or in AAC negative patients.

For analyzing an association between recorded comorbidities and NLR within the two groups, the variations were investigated using Dunn's multiple comparisons posttest, and Kruskal-Wallis test.

We further divided our patients into four subgroups depending on the presence or absence of each comorbid variable. Within the two groups of our study, for each recorded comorbidity we tested the median NLR differences. In the NLR association to SCAD we obtained statistically significant difference in the group 1 - SCAD absence (11 patients) versus group 2 - SCAD presence (10 patients), $\mathrm{p}=0.0397$. A significant $\mathrm{p}$ value $(\mathrm{p}=0.0339)$ was obtained as a result from the median differences of the NLR in the group 1 without concomitant LEAD (16 patients) versus group 2 without LEAD (fifteen patients). Median NLR values were higher statistically significant $(\mathrm{p}=0.0375)$ in aortic arch calcification group 1 patients with concomitant hypertensive heart disease compared to group 2 non hypertensive heart disease (10 patients).

Table I. Baseline characteristics, laboratory features of study group. Reference values of laboratory data are depicted.

\begin{tabular}{|c|c|c|c|c|}
\hline \multirow{2}{*}{ Parameters } & \multicolumn{2}{|c|}{ Patients (N) } & \multicolumn{2}{|c|}{ Mean \pm SD } \\
\hline & Group 1 & Group 2 & Group 1 & Group 2 \\
\hline Age (years) & 27 & 17 & $77.26 \pm 6.56$ & $65.82 \pm 5.39$ \\
\hline BMI (kg/m2) & 27 & 17 & $27.13 \pm 4.54$ & $29.93 \pm 4.50$ \\
\hline ESR $(5-17$ mm/h) & 13 & 6 & $22.54 \pm 16.28$ & $9.66 \pm 7.50$ \\
\hline Fibrinogen $(1.5-4.0 \mathrm{~g} / \mathrm{L})$ & 14 & 10 & $7.28 \pm 8.82$ & $4.82 \pm 0.37$ \\
\hline 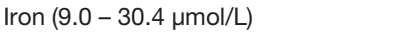 & 25 & 16 & $13.10 \pm 4.99$ & $14.20 \pm 5.54$ \\
\hline eGFR (mL/min/1.73 m2) & 27 & 17 & $48.04 \pm 10.65$ & $59.00 \pm 9.17$ \\
\hline Total cholesterol (2.8 - $5.2 \mathrm{mmol} / \mathrm{L})$ & 26 & 16 & $4.38 \pm 1.43$ & $4.34 \pm 1.16$ \\
\hline Triglycerides (0.55 - $1.90 \mathrm{mmol} / \mathrm{L})$ & 23 & 15 & $1.28 \pm 0.44$ & $1.38 \pm 1.11$ \\
\hline Alkaline phosphatase (100 - $300 \mathrm{U} / \mathrm{L})$ & 24 & 16 & $258.40 \pm 75.80$ & $200.30 \pm 50.16$ \\
\hline Uric acid (200 - $400 \mathrm{U} / \mathrm{L})$ & 27 & 16 & $383.60 \pm 105.20$ & $352.40 \pm 85.75$ \\
\hline Neutrophils $(1.5-7.5 \times 103 / \mu \mathrm{L})$ & 27 & 17 & $6.43 \pm 1.77$ & $3.86 \pm 1.24$ \\
\hline 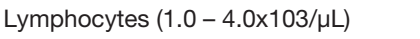 & 27 & 17 & $1.71 \pm 0.64$ & $1.52 \pm 0.38$ \\
\hline NLR & 27 & 17 & $4.33 \pm 2.47$ & $2.80 \pm 1.29$ \\
\hline
\end{tabular}

Abbreviations: BMI - body mass index; eGFR - estimated glomerular filtration rate (CKD-EPI formula); ESR - erythrocyte sedimentation rate; N - number; NLR - neutrophil-to-lymphocyte ratio; SD - standard deviation

Table II. Existing comorbidities of study group (44 patients).

\begin{tabular}{|c|c|c|c|c|c|c|}
\hline & \multicolumn{2}{|c|}{ Presence of SCAD } & \multicolumn{2}{|c|}{ LEAD diagnosis } & \multicolumn{2}{|c|}{ Established hypertensive heart disease } \\
\hline & YES & NO & YES & NO & YES & NO \\
\hline Group 1 (27 patients) & $16(59.26 \%)$ & $11(40.74 \%)$ & $11(40.74 \%)$ & $16(59.26 \%)$ & $10(37.03 \%)$ & $17(62.97 \%)$ \\
\hline Group 2 (17 patients) & $1(58.82 \%)$ & $7(41.18 \%)$ & $2(11.76 \%)$ & $15(88.24 \%)$ & $5(29.41 \%)$ & $12(70.59 \%)$ \\
\hline
\end{tabular}

Abbreviations: LEAD - lower extremity arterial disease; SCAD - stable coronary artery disease. Values are expressed in numbers and percentages. 
Table III. Comparison between presence of aortic arch calcification and baseline characteristics of the study groups (Mann-Whitney $\mathrm{U}$ test)

\begin{tabular}{|c|c|c|c|c|}
\hline Parameters & AAC & $\mathbf{N}$ & Median & $P$ value \\
\hline \multirow{2}{*}{ Age (years) } & Group 1 & 27 & 77.00 & \multirow{2}{*}{0.0001} \\
\hline & Group 2 & 17 & 66.00 & \\
\hline \multirow{2}{*}{ BMI (kg/m2) } & Group 1 & 27 & 26.00 & \multirow{2}{*}{0.0655} \\
\hline & Group 2 & 17 & 29.80 & \\
\hline \multirow{2}{*}{$\operatorname{ESR}(\mathrm{mm} / \mathrm{h})$} & Group 1 & 13 & 20.00 & \multirow{2}{*}{0.034} \\
\hline & Group 2 & 6 & 8.50 & \\
\hline \multirow{2}{*}{ Fibrinogen (g/L) } & Group 1 & 14 & 4.20 & \multirow{2}{*}{0.612} \\
\hline & Group 2 & 10 & 3.65 & \\
\hline \multirow{2}{*}{ Iron $(\mu \mathrm{mol} / \mathrm{L})$} & Group 1 & 25 & 13.50 & \multirow{2}{*}{0.5562} \\
\hline & Group 2 & 16 & 14.40 & \\
\hline \multirow{2}{*}{$\begin{array}{l}\text { eGFR }(\mathrm{mL} / \mathrm{min} / 1.73 \\
\mathrm{m} 2)\end{array}$} & Group 1 & 27 & 50.00 & \multirow{2}{*}{0.0007} \\
\hline & Group 2 & 17 & 56.00 & \\
\hline \multirow{2}{*}{$\begin{array}{l}\text { Total cholesterol } \\
(\mathrm{mmol} / \mathrm{L})\end{array}$} & Group 1 & 26 & 3.74 & \multirow{2}{*}{0.0074} \\
\hline & Group 2 & 16 & 4.15 & \\
\hline \multirow{2}{*}{ Triglyceride (mmol/L) } & Group 1 & 23 & 1.21 & \multirow{2}{*}{0.1554} \\
\hline & Group 2 & 15 & 0.95 & \\
\hline \multirow{2}{*}{$\begin{array}{l}\text { Alkaline phosphatase } \\
\text { (U/L) }\end{array}$} & Group 1 & 24 & 238.50 & \multirow{2}{*}{0.0074} \\
\hline & Group 2 & 16 & 190.50 & \\
\hline \multirow{2}{*}{ Uric acid (U/L) } & Group 1 & 27 & 381.00 & \multirow{2}{*}{0.3341} \\
\hline & Group 2 & 16 & 260.50 & \\
\hline
\end{tabular}

Abbreviations: AAC - aortic arch calcification; BMI - body mass index; ESR - erythrocyte sedimentation rate; eGFR - estimated glomerular filtration rate (CKD-EPI formula); $\mathrm{N}$ number of patients

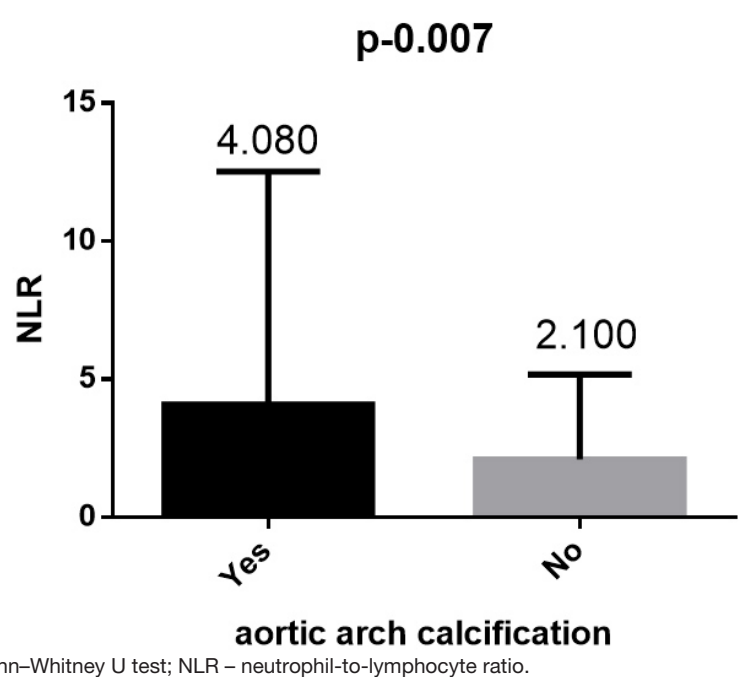

Fig. 1. Comparative analysis on neutrophil-to-lymphocyte ratio between the two groups

\section{Discussion}

Chronic kidney disease is currently a significant global health problem, since even with elimination of the generating factor it remains a progressive disease. Initial nephron loss induces adaptive mechanism characterised by hypertrophy and hyperfiltration in the remaining healthy nephrons with subsequent development of glomerular sclerosis characterised by proteinuria and hypertension $[36,37]$.

Table IV. Correlations between neutrophil-to-lymphocyte ratio and alkaline phosphatase, uric acid in the study group

\begin{tabular}{|c|c|c|c|c|c|c|c|}
\hline \multirow{2}{*}{ Parameters } & \multirow{2}{*}{$\begin{array}{c}\text { Spearman' } \\
\text { rho }\end{array}$} & \multicolumn{2}{|c|}{ NLR } & \multicolumn{2}{|c|}{ Alkaline phosphatase } & \multicolumn{2}{|c|}{ Uric acid } \\
\hline & & Group 1 & Group 2 & Group 1 & Group 2 & Group 1 & Group 2 \\
\hline \multirow{3}{*}{ NLR } & Correlation coefficient & 1.000 & 1.000 & 0.340 & -0.135 & 0.078 & 0.124 \\
\hline & Sig. (2-tailed) & 0 & 0 & 0.104 & 0.619 & 0.697 & 0.648 \\
\hline & $\mathrm{N}$ & 27 & 17 & 24 & 16 & 27 & 16 \\
\hline \multirow{3}{*}{$\begin{array}{l}\text { Alkaline } \\
\text { phosphatase }\end{array}$} & Correlation coefficient & 0.340 & -0.135 & 1.000 & 1.000 & 0.239 & -0.195 \\
\hline & Sig. (2-tailed) & 0.104 & 0.619 & 0 & 0 & 0.260 & 0.469 \\
\hline & $\mathrm{N}$ & 24 & 16 & 24 & 16 & 24 & 16 \\
\hline \multirow{3}{*}{ Uric acid } & Correlation coefficient & 0.078 & 0.124 & 0.239 & -0.195 & 1.000 & 1.000 \\
\hline & Sig. (2-tailed) & 0.697 & 0.648 & 0.260 & 0.469 & 0 & 0 \\
\hline & $\mathrm{N}$ & 27 & 16 & 24 & 16 & 27 & 16 \\
\hline
\end{tabular}

Abbreviations: NLR - neutrophil-to-lymphocyte ratio; N - number of patients; Sig. (2-tailed) - 2-Tailed statistical significance; Spearman's rho - Spearman's rank correlation coefficient

Table V. Correlations between neutrophil-to-lymphocyte ratio and fibrinogen, total calcium, total cholesterol, triglyceride of patients

\begin{tabular}{|c|c|c|c|c|c|c|c|c|c|c|c|}
\hline \multirow{2}{*}{ Parameter } & \multirow{2}{*}{$\begin{array}{c}\text { Spearman's } \\
\text { rho }\end{array}$} & \multicolumn{2}{|c|}{ NLR } & \multicolumn{2}{|c|}{ Fibrinogen } & \multicolumn{2}{|c|}{ Total calcium } & \multicolumn{2}{|c|}{ Total cholesterol } & \multicolumn{2}{|c|}{ Triglyceride } \\
\hline & & Group 1 & Group 2 & Group 1 & Group 2 & Group 1 & Group 2 & Group 1 & Group 2 & Group 1 & Group 2 \\
\hline \multirow{3}{*}{ NLR } & Correlation coefficient & 1.000 & 1.000 & 0.113 & 0.135 & 0.109 & 0.144 & 0.087 & -0.383 & 0.057 & -0.227 \\
\hline & Sig. (2-tailed) & 0 & 0 & 0.701 & 0.710 & 0.736 & 0.758 & 0.671 & 0.144 & 0.797 & 0.415 \\
\hline & $\mathrm{N}$ & 27 & 17 & 14 & 10 & 12 & 7 & 26 & 16 & 23 & 15 \\
\hline \multirow{3}{*}{ Fibrinogen } & Correlation coefficient & 0.113 & 0.135 & 1.000 & 1.000 & 0.143 & 0.232 & -0.517 & 0.287 & -0.282 & 0.239 \\
\hline & Sig. (2-tailed) & 0.701 & 0.710 & 0 & 0 & 0.787 & 0.658 & 0.070 & 0.421 & 0.329 & 0.507 \\
\hline & $\mathrm{N}$ & 14 & 10 & 14 & 10 & 6 & 6 & 13 & 10 & 14 & 10 \\
\hline \multirow{3}{*}{ Total calcium } & Correlation coefficient & 0.109 & 0.144 & 0.143 & 0.232 & 1.000 & 1.000 & 0.516 & 0.500 & 0.517 & -0.252 \\
\hline & Sig. (2-tailed) & 0.736 & 0.758 & 0.787 & 0.658 & 0 & 0 & 0.104 & 0.253 & 0.126 & 0.585 \\
\hline & $\mathrm{N}$ & 12 & 7 & 6 & 6 & 12 & 7 & 11 & 7 & 10 & 7 \\
\hline \multirow{3}{*}{$\begin{array}{l}\text { Total } \\
\text { cholesterol }\end{array}$} & Correlation coefficient & 0.087 & -0.383 & -0.517 & 0.287 & 0.516 & 0.500 & 1.000 & 1.000 & 0.399 & 0.243 \\
\hline & Sig. (2-tailed) & 0.671 & 0.144 & 0.070 & 0.421 & 0.104 & 0.253 & 0 & 0 & 0.066 & 0.383 \\
\hline & $\mathrm{N}$ & 26 & 16 & 13 & 10 & 11 & 7 & 26 & 16 & 22 & 15 \\
\hline \multirow{2}{*}{ Triglyceride } & Correlation coefficient & 0.057 & -0.227 & -0.282 & 0.239 & 0.517 & -0.252 & 0.399 & 0.243 & 1.000 & 1.000 \\
\hline & $\mathrm{N}$ & 23 & 15 & 14 & 10 & 10 & 7 & 22 & 15 & 23 & 15 \\
\hline
\end{tabular}

Abbreviations: NLR - neutrophil-to-lymphocyte ratio; N - number of patients; Sig. (2-tailed) - 2-Tailed statistical significance; Spearman's rho - Spearman's rank correlation coefficient 
When compared with the general population, patients with chronic kidney disease experience a more frequent and important cardiovascular disease, as the rate of cardiovascular events is inversely correlated to renal function, particularly for individuals in categories 4 and 5 of CKD. However, most patients do not progress to end-stage renal disease due to premature death as a consequence of cardiovascular complications [38]. Cardiovascular diseases related to renal impairment includes hypertension, coronary artery disease, heart failure, arrhythmias and lower extremity artery disease [39].

Early recognition and control of risk factors for atherosclerotic disease is mandatory in chronic kidney disease patients.

The occurrence of low level chronic inflammation during chronic kidney disease advancement is a determining factor for atherosclerosis progression.

Chronic kidney disease related inflammation bears a specific patho-mecanism that may enhance atherosclerosis through elevated generation of carbamylated low density lipoproteins, proinflammatory high density lipoproteins, cholesterol crystals, inflammasomes, and calciprotein particles $[34,40]$.

Neutrophil-to-lymphocyte ratio as a marker for inflammation can be an important tool in this context, as it has already emerged as an important marker for atherosclerotic disease, vascular calcification and also as a prognostic factor in CKD hypertensive patients $[15,18,27]$. Furthermore, neutrophil-to-lymphocyte ratio is a predictive factor for vascular calcification in end-stage renal disease [27].

Computation of neutrophil-to-lymphocyte ratio is a very simple mathematical and inexpensive method using results from automated white blood cell count when compared with assessment of any other inflammatory markers. This retrospective study identified a positive correlation between NLR and aortic arch calcification in hypertensive non end-stage CKD patients. Additionally, elevated alkaline phosphatase values and increased erythrocyte sedimentation rate had a positive association with aortic arch calcification; this is an expected result as alkaline phosphatase and erythrocyte sedimentation rate increase with ageing and fall in glomerular filtration rate. Our group with aortic arch calcification was comprised of older patients compared to the group without aortic arch calcification and had a more advanced renal disease.

Additionally our study has some limitations: is a small sample size single center retrospective study; routine phosphate - calcium characterization and intact serum parathormone levels (related to calcium metabolism) were not available. Regarding neutrophil-to-lymphocyte ratio distribution in the different comorbidities groups, we refrain from forming definite conclusions as a result of the limited number of patients included in the study.

To the best of our knowledge this is the first Romanian study which explores NLR as a novel inflammatory marker in hypertensive patients with less advanced CKD.

\section{Conclusions}

Neutrophil-to-lymphocyte ratio may be viewed as a risk factor for vascular calcification even in patients with less advanced kidney disease. The small number of individuals included in our study restrains us from reaching definite conclusions. Further prospective large scale studies, including primary care databases are mandatory in order to validate this accessible inflammatory marker as risk factor for arterial calcification in patients with CKD.

\section{Conflicts of Interest}

None to declare.

\section{Authors' contribution}

Claudia Floriana Suciu, MD, PhD student (Conceptualization; Data curation; Formal analysis; Investigation; Methodology; Supervision; Validation; Visualization; Writing -original draft; Writing - review \& editing) Andreea Varga, Lecturer, MD, PhD, MSc (Conceptualization; Data curation; Formal analysis; Investigation; Methodology; Supervision; Validation; Visualization; Writing - original draft; Writing - review \& editing)

Corneliu Florin Buicu, Assoc. Prof., MD, PhD (Conceptualization; Formal analysis; Methodology; Visualization; Writing - original draft; Writing - review \& editing) Ioan Tilea, Assoc. Prof, MD, PhD, MSc (Conceptualization; Formal analysis; Methodology; Validation; Visualization; Writing - original draft; Writing - review \&editing)

\section{References}

1. Fest J, Ruiter R, Ikram MA, Voortman T, van Eijck CHJ, Stricker BH. Reference values for white blood-cell-based inflammatory markers in the Rotterdam Study: a population-based prospective cohort study. Sci Rep. 2018;8(1):10566.

2. Stenvinkel P. Inflammation in end-stage renal disease--a fire that burns within. Contrib Nephrol. 2005;149:185-99.

3. Navarro JF, Milena FJ, Mora C, León C, García J. Renal pro-inflammatory cytokine gene expression in diabetic nephropathy: effect of angiotensinconverting enzyme inhibition and pentoxifylline administration. Am J Nephrol. 2006;26(6):562-70.

4. Fogo AB. Mechanisms of progression of chronic kidney disease. Pediatr Nephrol. 2007;22(12):2011-22.

5. Snaedal S, Heimbürger O, Qureshi AR, et al. Comorbidity and acute clinical events as determinants of C-reactive protein variation in hemodialysis patients: implications for patient survival. Am J Kidney Dis. 2009;53(6):1024-33.

6. Turkmen K, Guney I, Yerlikaya FH, Tonbul HZ. The relationship between neutrophil-to-lymphocyte ratio and inflammation in end-stage renal disease patients. Ren Fail. 2012;34(2):155-9.

7. Valente MJ, Rocha S, Coimbra S et al. Long Pentraxin 3 as a Broader Biomarker for Multiple Risk Factors in End-Stage Renal Disease: Association with All-Cause Mortality. Mediators Inflamm. 2019;3295725.

8. Krzanowski M, Krzanowska K, Gajda M, et al. Pentraxin 3 as a new indicator of cardiovascular-related death in patients with advanced chronic kidney disease. Pol Arch Intern Med. 2017;127(3):170-7.

9. Forget $P$, Khalifa C, Defour JP, et al. What is the normal value of the neutrophil-to-lymphocyte ratio? BMC Res Notes. 2017;10(1):12.

10. Templeton AJ, McNamara MG, Seruga B, et al. Prognostic role of neutrophil-to-lymphocyte ratio in solid tumors: a systematic review and meta-analysis. J Natl Cancer Inst. 2014;106(6):dju124.

11. Fest J, Ruiter TR, Groot Koerkamp B, et al. The neutrophil-to-lymphocyte ratio is associated with mortality in the general population: The Rotterdam Study. Eur J Epidemiol. 2019;34(5):463-79.

12. Christiansen $M H$, Barup $K \varnothing$, Samson MH. Neutrophil-lymphocyte-ratio distributions in a Danish population from general practice. Scand J Clin 
Lab Invest. 2019;79(1-2):75-79

13. Huguet E, Maccallini G, Pardini P, et al. Reference Values for Neutrophil to Lymphocyte Ratio (NLR), a Biomarker of Cardiovascular Risk, According to Age and Sex in a Latin American Population. Curr Probl Cardiol. 2019. pii: S0146-2806(19)30054-4.

14. Libby P. Inflammation in atherosclerosis. Arterioscler Thromb Vasc Biol. 2012;32(9):2045-51.

15. Corriere T, Di Marca S, Cataudella E et al. Neutrophil-to-Lymphocyte Ratio is a strong predictor of atherosclerotic carotid plaques in older adults. Nutr Metab Cardiovasc Dis. 2018;28(1):23-7.

16. Verdoia M, Barbieri L, Di Giovine G et al. Neutrophil to Lymphocyte Ratio and the Extent of Coronary Artery Disease: Results From a Large Cohort Study. Angiology. 2016;67(1):75-82.

17. Zhou S, Cai B, Zhang Y, Wang L, Liu X, Xu G. The Relationship between Neutrophil-to-Lymphocyte Ratio and Aortic Arch Calcification in Ischemic Stroke Patients. J Stroke Cerebrovasc Dis. 2017;26(6):1228-32.

18. Yoshitomi R, Nakayama M, Sakoh $T$ et al. High neutrophil/lymphocyte ratio is associated with poor renal outcomes in Japanese patients with chronic kidney disease. Ren Fail. 2019;41(1):238-43.

19. Yuan Q, Wang J, Peng Z et al. Neutrophil-to-lymphocyte ratio and incident end-stage renal disease in Chinese patients with chronic kidney disease: results from the Chinese Cohort Study of Chronic Kidney Disease (C-STRIDE). J TransI Med. 2019;17(1):86.

20. Mizobuchi M, Towler D, Slatopolsky E. Vascular calcification: The killer of patients with chronic kidney disease. J Am Soc Nephrol. 2009;20(7):1453-64.

21. London GM, Guerin AP, Marchais S, Metivier F, Pannier B, Adda H: Arterial media calcification in end-stage renal disease: Impact on all-cause and cardiovascular mortality. Nephrol Dial Transplant. 2003;18(9):1731- 40.

22. Neuen BL, Leather N, Greenwood AM, Gunnarsson R, Cho Y, Mantha ML. Neutrophil-lymphocyte ratio predicts cardiovascular and all-cause mortality in hemodialysis patients. Ren Fail. 2016;38(1):70-6.

23. lijima $K$, Hashimoto $H$, Hashimoto $M$ et al. Aortic arch calcification detectable on chest $\mathrm{X}$-ray is a strong independent predictor of cardiovascular events beyond traditional risk factors. Atherosclerosis. 2010;210(1):137-44.

24. Inoue T, Ogawa T, Ishida H, Ando $Y$, Nitta K. Aortic arch calcification evaluated on chest $X$-ray is a strong independent predictor of cardiovascular events in chronic hemodialysis patients. Heart Vessels. 2012;27(2):135-42.

25. Kalra SS, Shanahan CM. Vascular calcification and hypertension: cause and effect. Ann Med. 2012;44 Suppl 1:S85-92.

26. Zhou S, Cai B, Zhang Y, Wang L, Liu X, Xu G. The Relationship between Neutrophil-to-Lymphocyte Ratio and Aortic Arch Calcification in Ischemic Stroke Patients. J Stroke Cerebrovasc Dis. 2017;26(6):1228-32.

27. Turkmen K, Ozcicek F, Ozcicek A, Akbas EM, Erdur FM, Tonbul HZ. The relationship between neutrophil-to-lymphocyte ratio and vascular calcification in end-stage renal disease patients. Hemodial Int.
2014;18(1):47-53.

28. Cai K, Luo Q, Zhu B, et al. Neutrophil-lymphocyte ratio is associated with arterial stiffness in patients with peritoneal dialysis. BMC Nephrol. 2016;17(1):191.

29. Guo J, Fujiyoshi A, Willcox B et al. Increased Aortic Calcification Is Associated With Arterial Stiffness Progression in Multiethnic Middle-Aged Men. Hypertension. 2017;69(1):102-8.

30. Odink AE, Mattace-Raso FU, van der Lugt A et al. The association of arterial stiffness and arterial calcification: the Rotterdam study. J Hum Hypertens. 2008;22(3):205-7.

31. KDIGO 2012 Clinical Practice Guideline for the Evaluation and Management of Chronic Kidney Disease. Kidney Int Suppl. 2013;3(1)1150.

32. Williams B, Mancia G, Spiering W et al. 2018 ESC/ESH Guidelines for the management of arterial hypertension: The Task Force for the management of arterial hypertension of the European Society of Cardiology and the European Society of Hypertension: The Task Force for the management of arterial hypertension of the European Society of Cardiology and the European Society of Hypertension. J Hypertens. 2018;36(10):19532041.

33. Symeonidis G, Papanas N, Giannakis I et al. Gravity of aortic arch calcification as evaluated in adult Greek patients. Int Angiol. 2002;21:2336.

34. Task Force Members, Montalescot G, Sechtem U, Achenbach S et al. 2013 ESC guidelines on the management of stable coronary artery disease: the Task Force on the management of stable coronary artery disease of the European Society of Cardiology. Eur Heart J. 2013;34(38):2949-3003.

35. Aboyans V, Ricco JB, Bartelink MEL, et al. 2017 ESC Guidelines on the Diagnosis and Treatment of Peripheral Arterial Diseases, in collaboration with the European Society for Vascular Surgery (ESVS): Document covering atherosclerotic disease of extracranial carotid and vertebral, mesenteric, renal, upper and lower extremity arteries. Endorsed by: the European Stroke Organization (ESO), The Task Force for the Diagnosis and Treatment of Peripheral Arterial Diseases of the European Society of Cardiology (ESC) and of the European Society for Vascular Surgery (ESVS). Eur Heart J. 2018;39(9):763-816.

36. Yu HT. Progression of chronic renal failure. Arch Intern Med. 2003;163:1417-29.

37. Jacobson HR. Chronic renal failure: pathophysiology. Lancet. 1991. 338(8764):419-23.

38. Swaminathan S, Shah SV. Novel inflammatory mechanisms of accelerated atherosclerosis in kidney disease. Kidney Int. 2011;80(5):453-63.

39. Subbiah AK, Chhabra YK, Mahajan S. Cardiovascular disease in patients with chronic kidney disease: a neglected subgroup. Heart Asia. 2016;8(2):56-61.

40. Akchurin OM, Kaskel F. Update on inflammation in chronic kidney disease. Blood Purif. 2015;39(1-3):84-92. 